\title{
Method of Proofs in Mathematics: Higher Level Students' Understanding
}

\author{
Shiva Datta Dawadi \\ Sanothimi Campus, Sanothimi, Bhaktapur \\ Email: Shivadawadi7@gmail.com
}

\begin{abstract}
The objectives of this study are to determine students' understanding about different methods of proof in mathematics and compare their understanding with respect to their previous degree in education and other majors within the framework of a post-positivist research paradigm. Forty-two purposively selected students in the sample were selected. Their understanding of different methods of proof was determined with the help of a test paper consisting of 30 mathematical statements with proof. The study concluded that students are familiar with the methods of proofs used in verifying a mathematical statement. In addition, it is found that students with a previous degree in education and other majors differ in the level of understanding of different methods of proof in mathematics.
\end{abstract}

Keywords: Method of proofs, understanding, education, and non-education

\section{Background of the Study}

Mathematics largely deals with the collection of declarative statements. Whereas, we define a statement as a declarative sentence which is either true or false but not both without any ambiguity (Whitesitt, 2012). For example; 'two plus two equals four' and 'two plus two equals five' are both statements, the first because it is true and the second because it is false, in both cases we do not have any confusion/ambiguity to advocate its validity. But the mathematical sentence, ' $x+y>2$ ' is not a statement because the truth value of the statement depends on the values of $\mathrm{x}$ and $\mathrm{y}$ that the people have chosen. So in this regard, many mathematicians argue that mathematics is a pure isolated knowledge, which is useful because of its universal validity, value-free and culture-free which remains the same for all, regardless of time and context (Bishop et al., 2010). On the contrary, there are many mathematicians who continuously arguing that mathematical knowledge is corrigible and continually open to modification, its validity depends on the time and context (Ernest, 1991, p. 31). So, it is always a debatable issue among mathematician. As a human being, we always look for some logical inferences to argue certain knowledge or idea, which is called a proof (Solow, 2004). With the rapid change in time, technology and knowledge, various methods of proofs in mathematics are being emerged. Though the evolution of different methods of arguments opened the multiples ways of knowing and believing the mathematical concepts, it has raised confusion among students to select an appropriate method of proofs/arguments while establishing the logical argument for certain mathematical concepts. In this regard, the present study primarily aims to determine the understanding of different methods of proof of a mathematical statement among the students of master degree in mathematics education. 


\section{Statement of the Problem}

Selecting the best way of establishing the proof of a certain mathematical statement is always a subject of confusion. Unless and until we as well as our students are not clear about various methods of proof in mathematics, we neither can choose an appropriate method in a certain condition nor can suggest for the best selection. Recent studies showed that most of the graduate students in mathematics are not being felt comfortable in using various method of proof while proving a mathematical statement (Knuth, 2002). In this regard, determining the understanding of students' different methods of proof in mathematics is considered as the major problem of the study.

\section{Objective}

Following are the objectives of the study:

- To determine the students' understanding of different methods of proof in mathematics.

- To compare students' understanding of the methods of proof in mathematics with respect to their previous degree in education and other majors.

\section{Hypothesis}

For the second objective of the study, the following hypothesis was formulated:

- There is no significant difference in the understanding of methods of proof in mathematics with respect to education and other major backgrounds of students.

\section{Literature Review}

A proof is a sequence of logical statements, one implying another, which gives an explanation of why a given statement is true (Stefanowicz, 2014), describes simply the proof is a way of arguing the validity of statements based on some logical arguments. Olsker (2011) indicates the importance of mathematical proof saying, the mathematical proof lies at the foundations of mathematics. Rota (1997) describes mathematical proof as the proof of a mathematical statement is a sequence of logical steps which leads to the desired conclusion.

Every student throughout their educational careers is exposed to and participates in mathematical reasoning, justification, and proof (NCTM, 2000). The level and ways of mathematical proof also vary according to the academic level of the students as well as the mathematical communities they uncounted. For instance, the proof in early grade school is very different from that of a high school geometry class or other, the methods of proof in high school are different from that of undergraduate mathematics classes, and so on. This, in turn, may lead students to acquire a wide variety of beliefs about the criteria that make proofs valid and what constitutes a mathematical proof. These all contribute to students' familiarity with many techniques that can be used to prove the statements. It is important to notice that there is no one ideal proof, A theorem can be established using different techniques and none of them will be better or worse (Hersh, 1993).

Although there is a tendency of mathematicians to take proofs as taken for granted (Knuth, 2002) and following a 'definition-theorem-proof' format (Almeida, 2000). Ernst, 
Hodge, and Schultz (2015) suggest some remarkable critical lances for the mathematical community while writing mathematical proofs well; we must be skeptical consumers of our own work. We must learn how to look at our own writing as if it were written by others and at the same time ask the question within ourselves; Does it provide the correct level of detail?, Can I understand it line by line?

The two basic categorizations of mathematical proofs are direct proofs and indirect proofs. Direct proof assumes a given hypothesis or any other known statement, and then logically deduces a conclusion whereas, indirect proof also called the proof by contradiction, assumes the hypothesis together with the negation of the conclusion to reach the contradictory statement. It is often equivalent to proof by contra-positive.

In the present study, among various ways of establishing a mathematical proof for a mathematical statement some frequently used method of proofs are incorporated such as direct proof, proof by contradiction, proof by mathematical induction, method of proof by contrapositive, proof by experiment, and disprove by counter-example.

Let we discussed these all in brief:

\section{Direct Method}

Direct proof is probably the common approach to establish the validity of a mathematical statement which does not require an idea of any special techniques. The argument/conclusion is constructed using a series of connected simple statements one after another. To prove the given hypothesis, we may use axioms, as well as the previously established statements of different theorems. Propositions of the form $\mathrm{A} \Rightarrow \mathrm{B}$ are shown to be valid by starting at $\mathrm{A}$ by writing down what the hypothesis means and consequently approaching B using correct implications. We use the following general structures while proving mathematical statements through direct proof (Ferry, 2010):

- Express the statement to be proved in the form " $\forall x \in \mathrm{D}$, if $\mathrm{P}(\mathrm{x})$ then $\mathrm{Q}(\mathrm{x})$." Where $\mathrm{D}$ is a certain domain, $\mathrm{P}(\mathrm{x})$ is a hypothesis and $\mathrm{Q}(\mathrm{x})$ is the conclusion.

- Start the proof by supposing $\mathrm{x}$ is a particular but arbitrarily chosen element of $\mathrm{D}$ for which the hypothesis $\mathrm{P}(\mathrm{x})$ is true.

- Show that the conclusion $\mathrm{Q}(\mathrm{x})$ is true by using definitions, previously established results, and the rules for logical inference.

Example: Show that the sum of two consecutive whole numbers is odd.

Solution: Let $\mathrm{a}$ and $\mathrm{b}$ are two consecutive whole numbers

Here, $\mathrm{P}: \mathrm{a}$ and $\mathrm{b}$ are two consecutive whole numbers

$\mathrm{Q}: \mathrm{a}+\mathrm{b}$ is odd

Now,

$\mathrm{b}=\mathrm{a}+1$ [since $\mathrm{a}$ and $\mathrm{b}$ are consecutive whole numbers]

Then, $a+b=a+a+1=2 a+1$ (which is an odd)

Therefore PQ. Proved 


\section{Proof by Contradiction}

Proof by contradiction is a commonly used method of proof in mathematics. When trying to prove; statement $\mathrm{A} \Rightarrow$ statement $\mathrm{B}$ we take that the first statement (statement $\mathrm{A}$ ) is true but we assume the consequence statement (statement $\mathrm{B}$ ) is not true and try to deduce some contradictions. We follow the following general process to ague the proof by the method of contradiction (Roshen, 2012):

- Suppose the statement to be proved is false. That is, suppose that the negation of the statement is true.

- Show that this supposition leads logically to a contradiction.

Conclude that the statement to be proved is true

Example: Show that the sum of two consecutive integers' numbers is odd.

Proof: Assume $a$ and $b$ are consecutive integers

Assume that $(a+b)$ is not odd. If $(a+b)$ is not odd, then there is no integer $\mathrm{K}$ such that $(a+b)=2 k+1$

But, $\mathrm{a}+\mathrm{b}=\mathrm{a}+\mathrm{a}+1=2 \mathrm{a}+1$. This is the contradiction. Hence, by contradiction, the sum of two integers is odd.

\section{Proof by Mathematical Induction}

While proving the mathematical statement by mathematical induction, we show the statement is true for $n=1$ or also for other values of $n$ greater than 1 . Then we suppose the statement is true for $\mathrm{n}=\mathrm{k}$ (some positive natural number). Then, on that basis, we show that the statement is true for $n=k+1$. Finally, we conclude that the statement is true for all values of $n$ N.

The principle of mathematical induction states that if $\mathrm{P}(\mathrm{n})$ be the mathematical induction and if

i) $\quad \mathrm{P}(1)$ is true

ii) $\quad \mathrm{P}(\mathrm{k}+1)$ is true whenever $\mathrm{P}(\mathrm{k})$ is true

Then $\mathrm{P}(\mathrm{n})$ is true for all $\mathrm{n} \mathrm{N}$.

While proving mathematical statements using the principle of mathematical induction, first we suppose mathematical statement as $\mathrm{P}(\mathrm{n})$. We use two main steps, the basis step, and the inductive step. In basis step, we show $\mathrm{P}(1)$ is true. In inductive step, we assume $\mathrm{p}(\mathrm{k})$ is true for some positive integer value of $n$, and using it we finally show $\mathrm{P}(\mathrm{k}+1)$ is true. And, we conclude the mathematical statement $\mathrm{P}(\mathrm{n})$ is true for all $\mathrm{nN}$.

Example: Prove that the product of two consecutive natural numbers is even.

Proof: Let $P(n)=n(n+1)$, where $n$ and $n+1$ are two consecutive natural numbers.

Step 1(Basis step): Take $\mathrm{n}=1$ then $\mathrm{P}(1)=1(1+1)=2$ (true, because it is an even number)

Step 2(Inductive step): Let $\mathrm{p}(\mathrm{k})$ is even for $\mathrm{n}=\mathrm{k}$, i.e. $\mathrm{P}(\mathrm{k})=\mathrm{k}(\mathrm{k}+1)$ is even

Now, let us take $\mathrm{n}=\mathrm{k}+1$, then

$\mathrm{P}(\mathrm{k}+1)=(\mathrm{k}+1)(\mathrm{k}+1+1)=(\mathrm{k}+1)(\mathrm{k}+2)$ 
Since $(\mathrm{k}+1)$ is even, $(\mathrm{k}+1)(\mathrm{k}+2)$ is also even because even times any other positive integer is always even.

Therefore, $\mathrm{p}(\mathrm{k}+1)$ is even.

Hence by the principle of mathematical induction, we conclude that the product of any two consecutive natural numbers is even.

\section{Method of Proof by Contrapositive}

Proof by contra-positive can be considered as a type of proof by contradiction. We start the proof of the mathematical statements in the same way in both cases as the opposite of the statement. So, while verifying the statement A gives the statement B, we assume "not B", but in this method we argue to turn up at "not A" because of assuming not A. The whole process indicates the fact that the statements " $\mathrm{A} \Rightarrow \mathrm{B}$ " is equivalent to "not $\mathrm{B} \Rightarrow$ not $\mathrm{A}$ ". The following general procedure can be used to prove a mathematical statement by the method of contrapositive.

- Express the statement to be proved in form $\forall x$ in $\mathrm{D}$ (domain), if $\mathrm{P}(\mathrm{x})$ then $\mathrm{Q}(\mathrm{x})$.

- Write the statement in the contra-positive form as $\forall x$ in $\mathrm{D}$, if $\mathrm{Q}(\mathrm{x})$ is false then $\mathrm{P}(\mathrm{x})$ is false.

- We prove by the contra-positive statement by direct proof. And, we conclude that the statement A gives/implies statement B.

Example: Show that the sum of two consecutive numbers is odd.

Proof: We want to show,

If $\mathrm{a}$ and $\mathrm{b}$ are consecutive integers then the sum $\mathrm{a}+\mathrm{b}$ is odd.

Here, $P$ : If $a$ and $b$ are consecutive integers

Q: $a+b$ is odd.

Its contrapositive statement is,

If the $\operatorname{sum}(\mathrm{a}+\mathrm{b})$ is not odd, then $\mathrm{a}$ and $\mathrm{b}$ are not consecutive integers.

i.e Q: $(a+b)$ is not odd

$\mathrm{P}: \mathrm{a}$ and $\mathrm{b}$ are not consecutive integers.

$\mathrm{Q}$ and $\mathrm{P}$ are the negation of the statements $\mathrm{Q}$ and $\mathrm{P}$.

Assume that, $\operatorname{sum}(\mathrm{a}+\mathrm{b})$ is not odd. That means

So, $a+b=2 k+1=k+(k+1)$ does not hold for any integer.

But, $\mathrm{k}+1$ is the successor of $\mathrm{k}$, this implies a and $\mathrm{b}$ cannot be consecutive.

Here we have shown QP

Therefore, PQ

\section{Disprove by Counterexample}

While proving by the use counter example, we seek for at least on counter-example in which the given statement is true but the conclusion is false. 
To disprove a statement of the form if A then B for every element in a certain domain, find a value of $\mathrm{x}$ in the domain for which the hypothesis $\mathrm{A}(\mathrm{x})$ is true and the conclusion $\mathrm{B}(\mathrm{x})$ is false. Such particular $\mathrm{x}$ is called a counter example to disprove if $\mathrm{A}(\mathrm{x})$ then $\mathrm{B}(\mathrm{x})$. It is interesting that one counterexample is enough to say that the statement is not true, even though there will be many examples in its favor (Ferry, 2010)

Example: Disprove the following statement: $\forall$ real numbers $a$ and $b$, if $a^{2}=b^{2}$ then $a=b$.

Solution: Here, $\mathrm{a}^{2}=\mathrm{b}^{2}$

Take $\mathrm{a}=-13$, then $\mathrm{a}^{2}=(-13)^{2}=169$

Take $b=13$, then $b^{2}=13^{2}=169$

In this example $\mathrm{a}^{2}=\mathrm{b}^{2}$ but $\mathrm{ab}$

\section{Proof by the Method of Experiment}

The poof of mathematical statements by experimenting the given statement in the given domain is called the method of experiment, when the domain is finite or only the finite number of elements satisfies the given condition then we can check the validity of the given statements by the direct experimentation.

Example: Prove that, for every $\mathrm{nZ}$, if $\mathrm{n}$ is even and $7 \leq \mathrm{n} \leq 17$, then $\mathrm{n}$ can be written as a sum to two prime numbers.

Solution: Even numbers between 7 and 27 are 8, 10, 12, 14, and 16.

Now, let we check one by one;

Experiment I: $\quad 8=5+3$

Experiment II: $\quad 10=5+5$

Experiment III: $\quad 12=7+5$

Experiment IV: $\quad 14=7+7$

Experiment V: $\quad 16=11+5$

From these experiments, we can conclude that even numbers between 7 and 17 can be written as a sum of two prime numbers.

Finally, with an intention to determine the understanding of graduate (master degree) students regarding the various methods of proofs in mathematics, the present study has been conducted.

\section{Methodology}

The present study was conducted within the framework of the post-positivist research paradigm. The post- positivistic research aims to produce objective and generalizable knowledge about social patterns, seeking to affirm the presence of universal properties/laws in relationships amongst pre-defined variables (Taylor, 2013), the present study with an aim to identify the knowledge of the graduate students' regarding different method of proof for the mathematical statements was conducted with this framework based on quantitative research design. Forty-two students who were studying for a master degree in mathematics education were taken as the sample of the study. 


\section{Sample of the Study}

Forty-two students majoring mathematics education in master degree were selected purposefully for the study, out of those forty-two students, 27 students were completed their Bachelor study in education whereas 15 students were completed their Bachelor from other streams.

\section{Data Collection Tools}

With an objective to determine students' understanding of various methods of proof of mathematical statements in mathematics, a test paper was constructed consisting of thirty different mathematical statements (theorems and examples) with their proofs. The proofs were based on the literature mentioned above as direct proof, proof by contradiction, proof by mathematical induction, proof by contra-positive, proof by experiment, disprove by counterexample. Each of these six categories (method of proofs) consists of five examples as proof of the statements. The theorems and examples are based on the basic concepts of Algebra and Number theory to which the samples students were familiar in their undergraduate studies.

\section{Ethical Concern}

In the process of research, the researcher tried to address the ethical issues from the various points of view as:

- First, the researcher made clear about the purpose and process of the research to the respondents so that they will decide whether to participate or not.

- Then the researcher ensured that the research will not harm in any way to the respondents.

- The respondents were informed that they will have the right to refuse or terminate their participation at any stage of the research.

\section{Data Analysis and Interpretation}

After preparing the test paper, it was given to the sampled students and they were asked to identify the particular type of method of proof in the proof of each of the given statements. And, their responses in each statement were considered as the data for the study. The collected data were analyzed and interpreted with the help of different descriptive and inferential statistical tools (frequency, mean and t-test).

\section{Students Understanding of Different Methods of Proofs}

As there were thirty statements with their proofs into six different categories as direct proof, proof by contradiction, proof by mathematical induction, proof by contra-positive, proof by experiment, and disprove by counterexample, there were five examples in each of those methods of proof. And, as there were forty-two students in the sample, each category consisted of 210 responses. The right and wrong responses of the teachers were organized in a table and on the basis of this table, students understanding of different methods of proof in mathematics were analyzed and discussed. 
Table I

Identification of methods of proof by the sampled students

\begin{tabular}{lllll}
\hline Categories & $\begin{array}{l}\text { No. of correct } \\
\text { responses }\end{array}$ & $\begin{array}{l}\text { \% of correct } \\
\text { responses }\end{array}$ & $\begin{array}{l}\text { No. of wrong } \\
\text { responses }\end{array}$ & $\begin{array}{l}\text { \% of correct } \\
\text { responses }\end{array}$ \\
\hline Direct proof & 182 & 86.66 & 28 & 13.33 \\
\hline Proof by contradiction & 163 & 64.76 & 47 & 35.23 \\
\hline $\begin{array}{l}\text { Proof by mathematical } \\
\text { induction }\end{array}$ & 204 & 97.14 & 6 & 2.86 \\
\hline $\begin{array}{l}\text { Proof by contrapositive } \\
\text { Disprove by counter }\end{array}$ & 146 & 45.71 & 114 & 54.28 \\
\hline $\begin{array}{l}\text { example } \\
\text { Proof by experiment }\end{array}$ & 180 & 69.52 & 64 & 30.47 \\
\hline
\end{tabular}

Table I shows that most of the students were familiar with the concept of methods of proof used in verifying a mathematical statement. As shown in the table, the most well-known method of proof among those six categories was the proof by the method of induction as $97.14 \%$ sampled students answered correctly whereas 'proof by contra-positive' is found more confusing among students in comparison to other methods of proof.

\section{Comparison of students' knowledge about different methods of proofs based on their previous degree (education and non-education)}

The following Table II shows the comparison of students' understanding of different methods of proof between the group of students based on education and other majors background.

Table II

Comparison of knowledge in methods of proof of students based on their previous degree

\begin{tabular}{lllllll}
\hline Backgrounds & No. of Students & Mean & S.D. & d.f. & t-value & Decision \\
\hline Education & 27 & 26 & 2.04 & 40 & 2.504 & $\mathrm{H}_{0}$ is rejected \\
\hline Other Majors & 15 & 21 & 3.16 & & & \\
\hline
\end{tabular}

The table illustrates that there was a significant difference (at 0.05 level of significance) in students' understanding of the different method of proof between the group of students based on the previous degree in education and other major which means the understanding of students regarding the different method of proof in mathematics is affected by the background of the students as education or other majors. 


\section{Findings and Discussion}

Based on the above-mentioned analysis and interpretation, it was found that graduatelevel students in mathematics education are familiar with most of the commonly practiced methods of proofs used in verifying different mathematical statements. They can understand and apply the proof of one statement into the proof of another statement (Bass, 2009). As Mason (1982) viewed, proving as convincing oneself, convincing a friend, and convincing an enemy. Though students were familiar with various methods, the degree of their understanding varies. Majority of the students were found to be familiar with the method of mathematical induction, whereas many students had difficulty in identifying the method of proof by contrapositive. These findings were also supported by Hanna (2000) as this type of method was easy to identify and follow a fixed structure. It was also interesting to find that the background of students (education or non-education) is also affected their understanding of different methods of proofs for the mathematical statements. There was a significant difference in the average score of the students in the test paper based on education and other majors backgrounds. The average performance of the students from the education background was higher than the group of students without an education background. Thus it was found that students from education backgrounds were more familiar with various methods of proof in mathematics. Though students were a bit confused while deciding a certain method of proof in a certain condition, it created a locus of logical-mathematical debate (Villiers, 1990). The study hopefully be benefited to the students to be familiar with an idea of different methods of proof as well as to know their level of understanding regarding different methods of proof for a mathematical statements whereas it is beneficial to the teachers to know about the method of proof at which the majority of the students feel confusion so that the teacher may emphasize more on that.

\section{Conclusion}

With the help of the above-mentioned analyses, findings, and discussion, it was concluded that graduate students in mathematics education in Nepal are familiar with different methods of proof used in various mathematical statements. And, also the level of understanding about different methods of proof differed by their previous academic stream (as education or other majors). On the basis of those findings, students from other majors need more support in identifying the best methods of proof in verifying mathematical statements.

\section{Reference}

Almeida, D. (2000). A survey of mathematics undergraduates' interaction with proof: Some implications for mathematics education. International Journal of Mathematics Education, Science and Technology, 31(6), 869-890.

Bass, H. (2009). How do you know that you know? Making believe in mathematics. Retrieved from: http://deepblue.lib.umich.edu/handle/2027.42/64280

Bishop, A.,Clements, M.A., Keitel, C., Kilpatrick, J. Laborde, A .(2010). International Handbook of Mathematics Education. Springer publication

Ernst, D. C., Hodge, A., \& Schultz, A. (2015). Enhancing proof writing via cross-institutional peer review. Primus, 25(2), 121-130.

Mathematics Education Forum Chitwan, September 2019, Issue 4, Year 4 
Ferry, D. (2010). Methods of Proofs. (Retrieved from Google)

Hanna, G. (2000). Proof, explanation and exploration: An overview. Educational Studies in Mathematics, 44 (1-2), 5-23.

Hersh, R. (1993). Proving is convincing and explaining. Educational Studies in Mathematics, 24(4), 389-399

Knuth, E. J. (2002). Secondary school mathematics teachers' conceptions of proof. Journal for Research in Mathematics Education, 33(5):379- 405.

Mason, J. \& L. and Stacey, K. (1982).Thinking mathematically. Wokingham: Addison-Wesley Publishing Company.

NCTM (2000). Principles and standards for school mathematics. NCTM, Reston, VA.

Olsker, T. C. (2011). What do we mean by mathematical proof? Department of Mathematics: California State University Fullerton.

Rosen, K. H., (2012). Discrete Mathematics and its Application. Monmouth University McGraw-Hill Companies, Inc.: NewYork

Rota, G. C. (1997). The phenomenology of mathematical proof. Journal of Automated Reasoning 23(3-4), 197-234.

Solow, D. (2004). How to read and do proofs: An introduction to mathematical thought. Orlando, Florida: Academic Press.

Stefanowicz, A.(2014). Proofs and Mathematical Reasoning.Teaching and Teacher Education, $17(1), 15-31$.

Villiers, M. D., (1990). The role and function of proof in mathematics. Pythagoras, 24, 17-24

Whitesitt, E. J. (2012). Boolean Algebra and Its Application. Dover Publication. INC. New York: USA 